Supporting Information

\title{
Ultrafast Absorption of Polysulfides through Electrostatic Confinement by Protonated Molecule for Highly Efficient Li-S Battery
}

Hongfei Bao, ${ }^{\dagger}$ ll Yujiao Dong, ${ }^{\dagger}$ "ll Xuesong Wu, ${ }^{\S}$ Guangfu Li, ${ }^{\dagger}$ Fulong Zhu, ${ }^{\dagger}$ Wei Guan, ${ }^{*}+\dot{\dagger}$ Xin-long Wang ${ }^{*}+\dot{\dagger}$ and Zhong-min Su ${ }^{*,+}$

†National \& Local United Engineering Laboratory for Power Battery, Key Laboratory of Polyoxometalate Science, Institute of Functional Material Chemistry, Northeast Normal University, Changchun, Jilin, 130024, P. R. China

$\S$ Jilin Provincial Science and Technology Innovation Center of Optical Materials and Chemistry, School of Chemistry and Environmental Engineering, Changchun University of Science and Technology, Changchun, 130022 China

*E-mail: guanw580@nenu.edu.cn,wangx1824@nenu.edu.cn and zmsu@nenu.edu.cn. 
Chemical: 1,4-butanediamine $\quad\left(\mathrm{C}_{4} \mathrm{H}_{14} \mathrm{~N}_{2}, \quad\right.$ Aladdin Reagent, AR $), 3-$ pyridinecarboxaldehyde $\left(\mathrm{C}_{6} \mathrm{H}_{5} \mathrm{NO}\right.$, Aladdin Reagent, AR), sodium borohydride ( $\mathrm{NaHB}_{4}$, Fuchen Reagent, AR), sodium hydroxide (NaOH, Fuchen Reagent, AR), dichloromethane $\left(\mathrm{CH}_{2} \mathrm{Cl}_{2}\right.$, Aladdin Reagent, $\left.\mathrm{AR}\right)$, nitric acid $\left(\mathrm{HNO}_{3}\right.$, Aladdin Reagent, AR), 1,3-dioxolane (DOL, Sigma-Aldrich, AR), 1,2-dimethoxyethane (DME, Sigma-Aldrich, AR), lithium bis(trifluoromethanesulfon)imide (LiTFSI, Alfa Aesar), lithium nitrate $\left(\mathrm{LiNO}_{3}\right.$, Aladdin Reagent, AR), lithium-sulfur $\left(\mathrm{Li}_{2} \mathrm{~S}\right.$, Alfa Aesar, AR), sulfur (S, sinopharm Chemical Reagent Co. Ltd., AR), poly(vinylidene fluoride) (PVDF, Solef@5130, Solvay), N-methyl-2-pyrrolidone (NMP, Aladdin Reagent, AR), 1,1'-Dibenzyl-4,4'-bipyridinium Dichloride Hydrate (DBDH, Ark Pharm, Inc.).

Characterizations: ${ }^{1} \mathrm{H}$ NMR spectra were recorded on Bruker $600 \mathrm{MHz}$ instrument. Thermogravimetric analysis (TGA) was performed on a PerkinElmer TG-7 analyzer over the temperature $20-600{ }^{\circ} \mathrm{C}$ in a nitrogen-gas atmosphere with a heating rate of $10{ }^{\circ} \mathrm{C} \mathrm{min}^{-1}$. Powder X-ray diffraction (XRD) measurements were recorded on a Rigaku/Dmax 2200 pc diffractometer with $\mathrm{Cu}-\mathrm{K} \alpha(\lambda=1.5418 \AA)$ radiation in the range $5-80^{\circ}$. The Raman spectra of samples were recorded by Raman spectrometer (JY HR-800, HORIBA JOBIN YVON) with an excitation laser beam wavelength of $633 \mathrm{~nm}$. TEM was conducted on the JEM-2100 microscopes at $200 \mathrm{kV}$. The UV-Vis spectra were carried out in the $200-800 \mathrm{~nm}$ spectral range by a Cary $500 \mathrm{UV}-\mathrm{Vis}$ spectrophotometer. XPS studies were performed to research the composition and electronic state of the $\mathrm{Li}_{2} \mathrm{~S}_{\mathrm{n}},\left[\mathrm{H}_{2} \mathrm{PBD}\right]^{2+} \cdot\left(\mathrm{NO}_{3}\right)_{2}{ }^{2-}$ and $\mathrm{H}_{2} \mathrm{PBD}-\mathrm{S}_{\mathrm{n}}($ ESCALAB 250, Thermo). 


\section{Supplementary Figures}

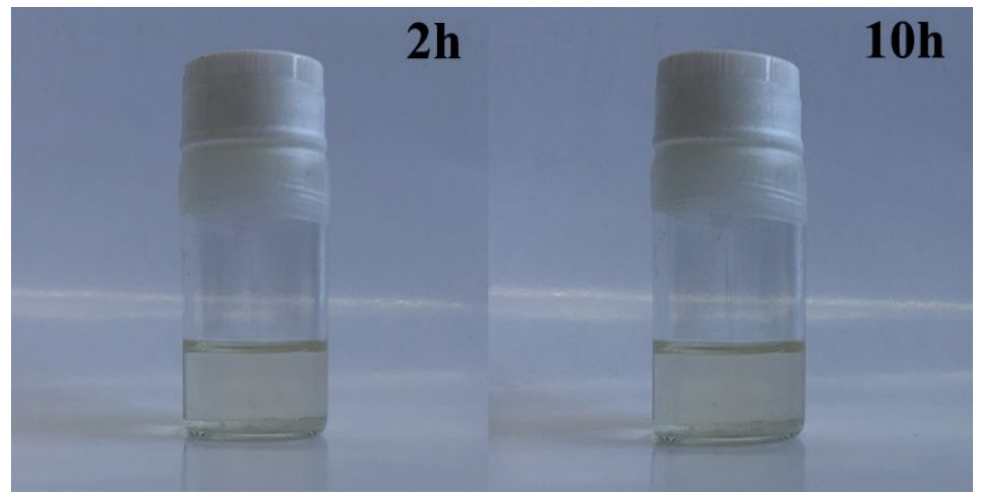

Figure S1. The solubility experiment of the $\left[\mathrm{H}_{2} \mathrm{PBD}\right]^{2+} \cdot\left(\mathrm{NO}_{3}\right)_{2}{ }^{2-}$ in the electrolyte for 2 and 10 hours, which proves that the $\left[\mathrm{H}_{2} \mathrm{PBD}\right]^{2+} \cdot\left(\mathrm{NO}_{3}\right)_{2}{ }^{2-}$ is insoluble and stable in the electrolyte. 


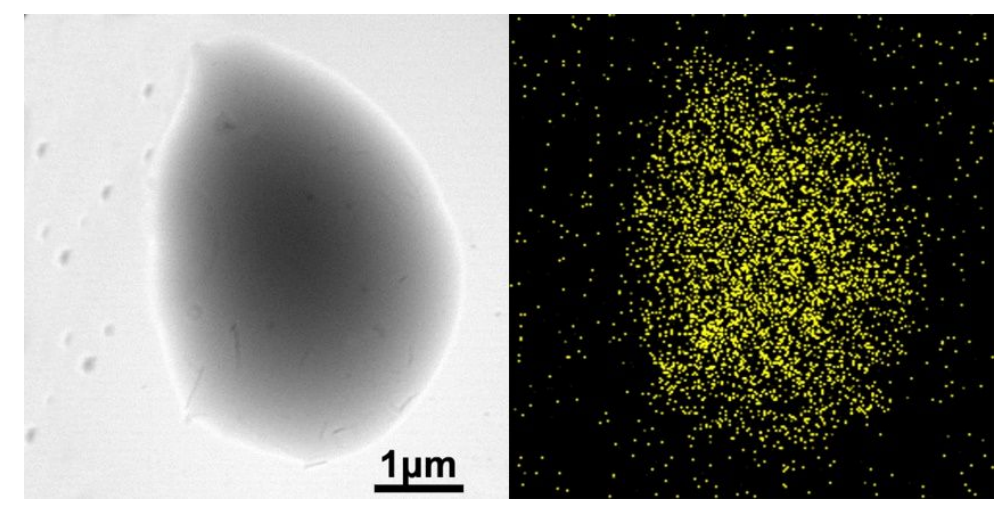

Figure S2. TEM image and the sulfur elemental mapping for $\mathrm{H}_{2} \mathrm{PBD}-\mathrm{S}_{\mathrm{n}}$. 


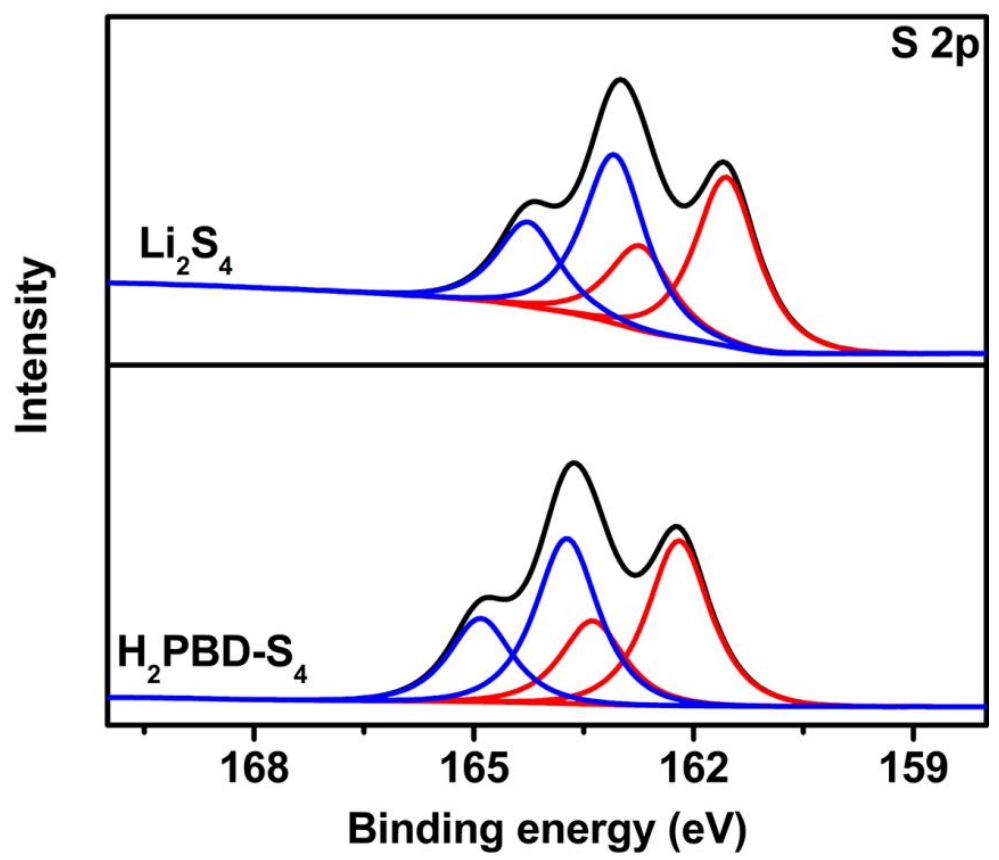

Figure S3. S $2 p$ XPS spectra of $\mathrm{Li}_{2} \mathrm{~S}_{4}$ and $\mathrm{H}_{2} \mathrm{PBD}-\mathrm{S}_{4}$. 


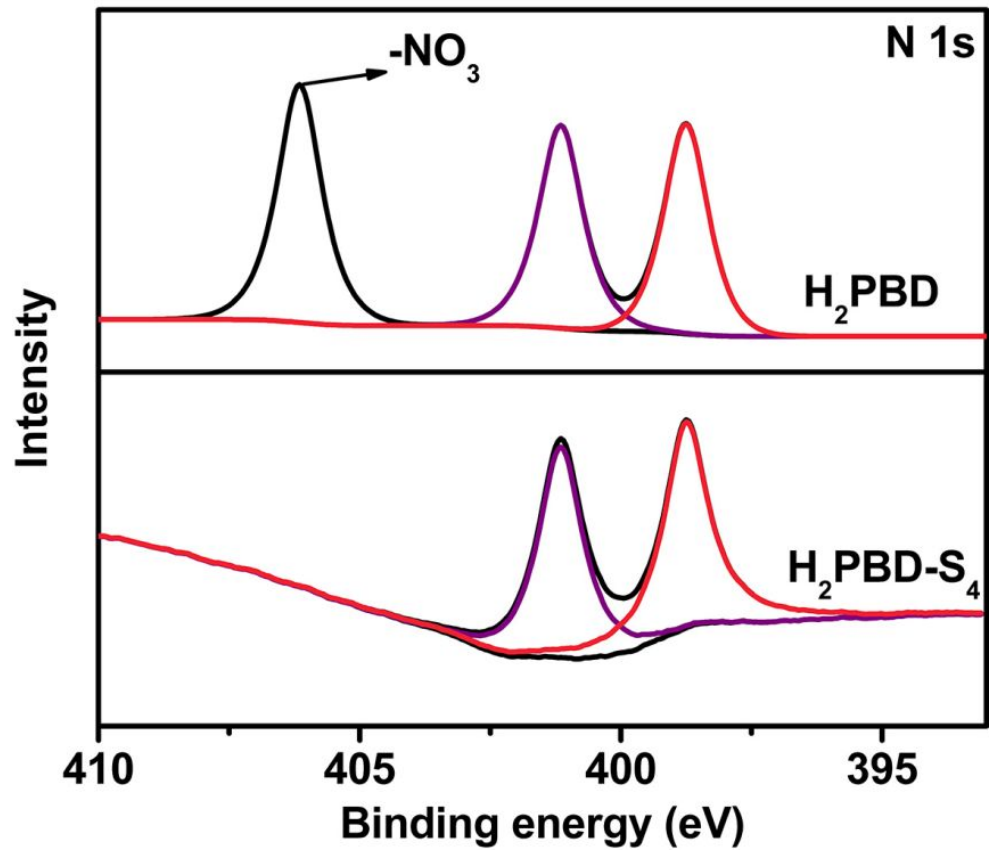

Figure S4. N 1s XPS spectra of $\left[\mathrm{H}_{2} \mathrm{PBD}\right]^{2+} \cdot\left(\mathrm{NO}_{3}\right)_{2}{ }^{2-}$ and $\mathrm{H}_{2} \mathrm{PBD}-\mathrm{S}_{4} \cdot{ }^{[1]}$ 


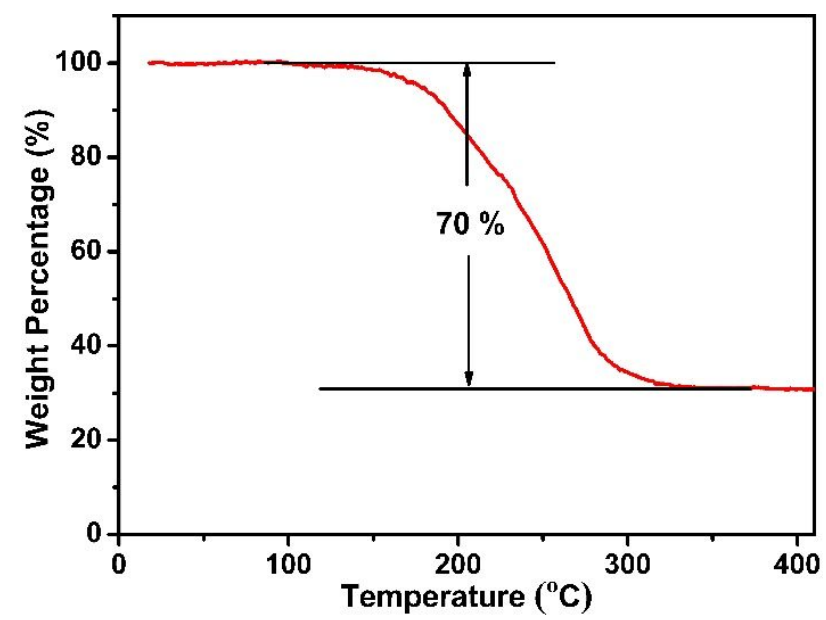

Figure S5. TGA of G/S composites. 


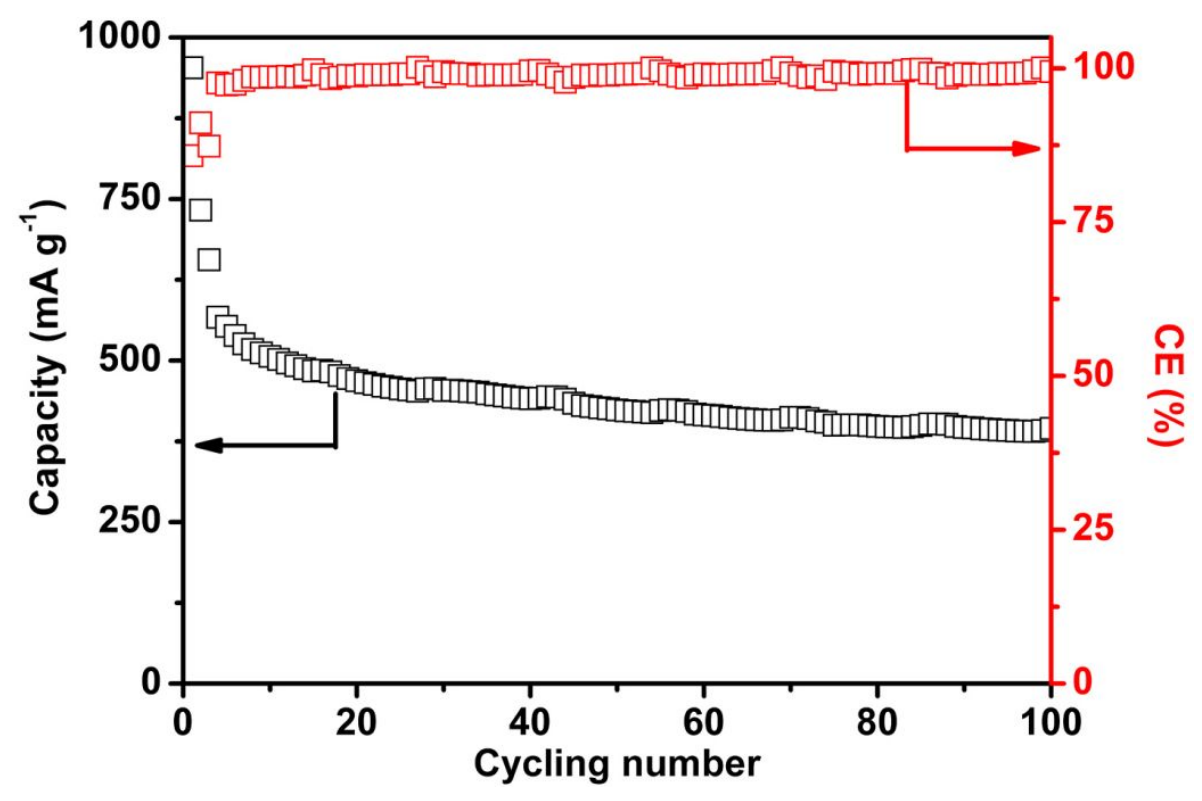

Figure S6. High sulfur loading $\left(8 \mathrm{mg} \mathrm{cm}^{-2}\right)$ and low electrolyte content $\left(10 \mu \mathrm{L} \mathrm{mg}^{-1}\right)$ of $\left[\mathrm{H}_{2} \mathrm{PBD}\right]^{2+} \cdot\left(\mathrm{NO}_{3}\right)_{2}{ }^{2-} / \mathrm{CNT} / \mathrm{S}$ electrode at $0.5 \mathrm{C}$. 


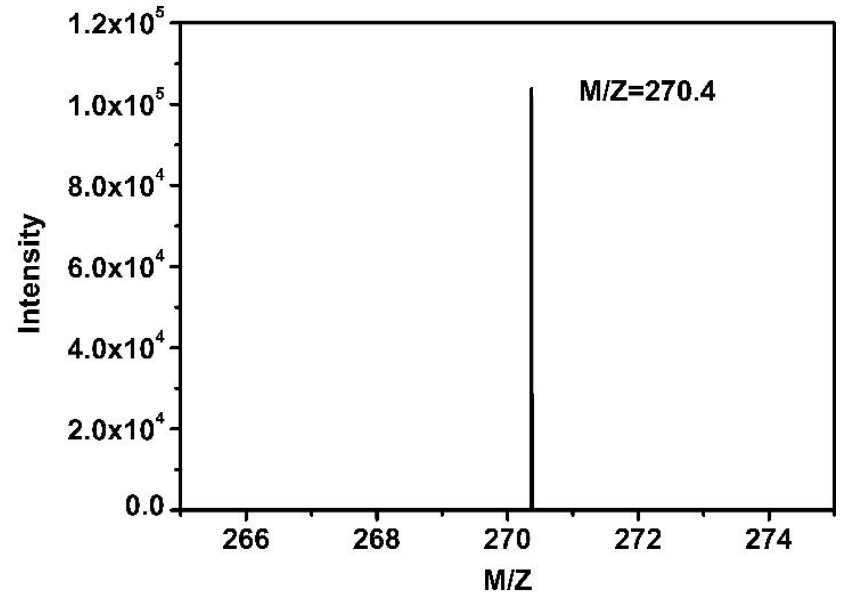

Figure S7. Mass spectra of PBD. 


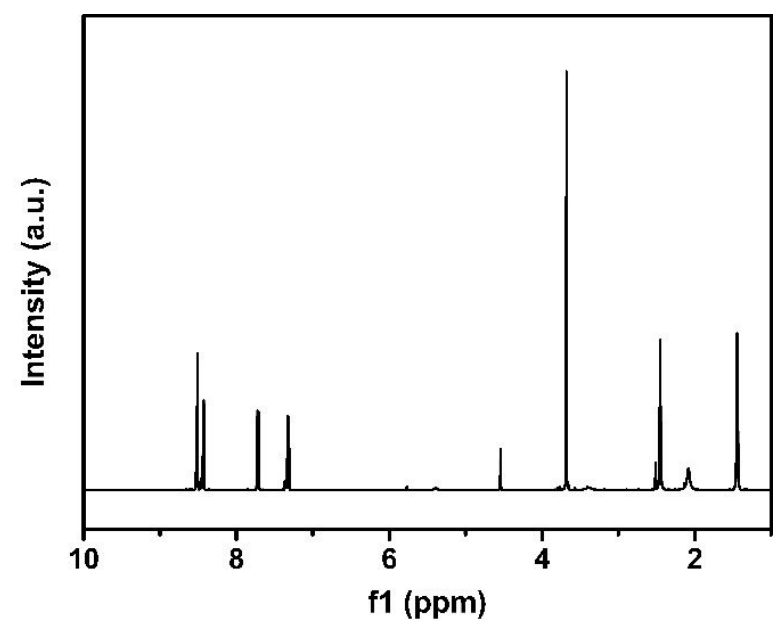

Figure S8. NMR spectra of PBD. 


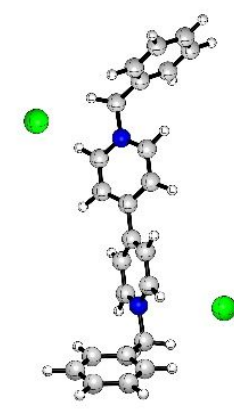

DBDH

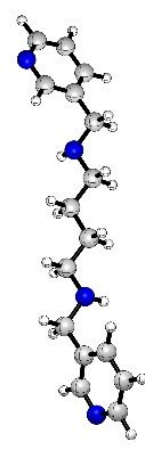

PBD

Figure S9. The Models of PBD, DBDH molecule. The grey, white, blue and green color refer to carbon, hydrogen, nitrogen and chlorine, respectively. 


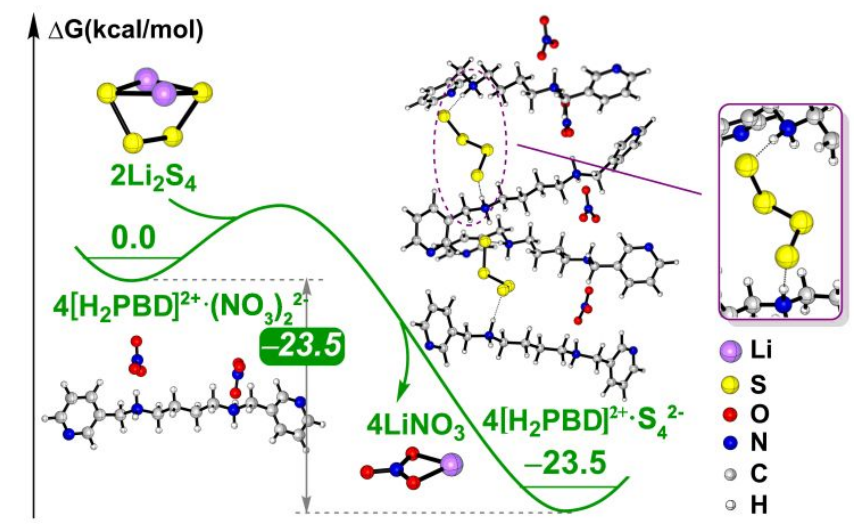

Figure S10. The Gibbs free energy change $(\Delta \mathrm{G})$ of ion exchange reaction between $\mathrm{Li}_{2} \mathrm{~S}_{4}$ and $\left[\mathrm{H}_{2} \mathrm{PBD}\right]^{2+} \cdot\left(\mathrm{NO}_{3}\right)_{2}{ }^{2-}$ calculated at the $\mathrm{SMD}(\mathrm{DME}) / \omega \mathrm{B} 97 \mathrm{XD} / 6-311 \mathrm{G}(\mathrm{d}, \mathrm{p})$ level. 

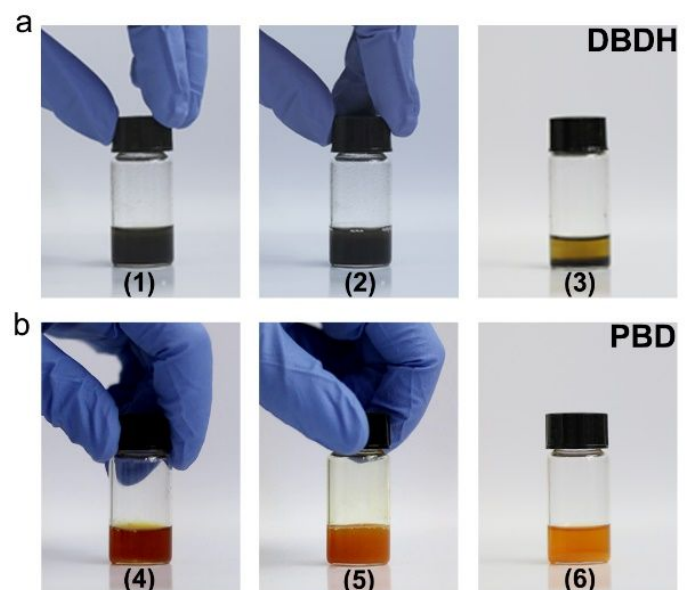

PBD

shake 20s

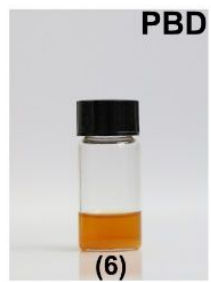

Figure S11. The digital photos of (a) DBDH and (b) PBD rapid adsorption processes. (1), (4) were the state before the rapid shaking; (2), (5) were the state after the shaking 20 seconds; (3), (6) were the state after 1 minute of stillness. The DBDH has more rapid adsorption capacity to PSs than PBD. 


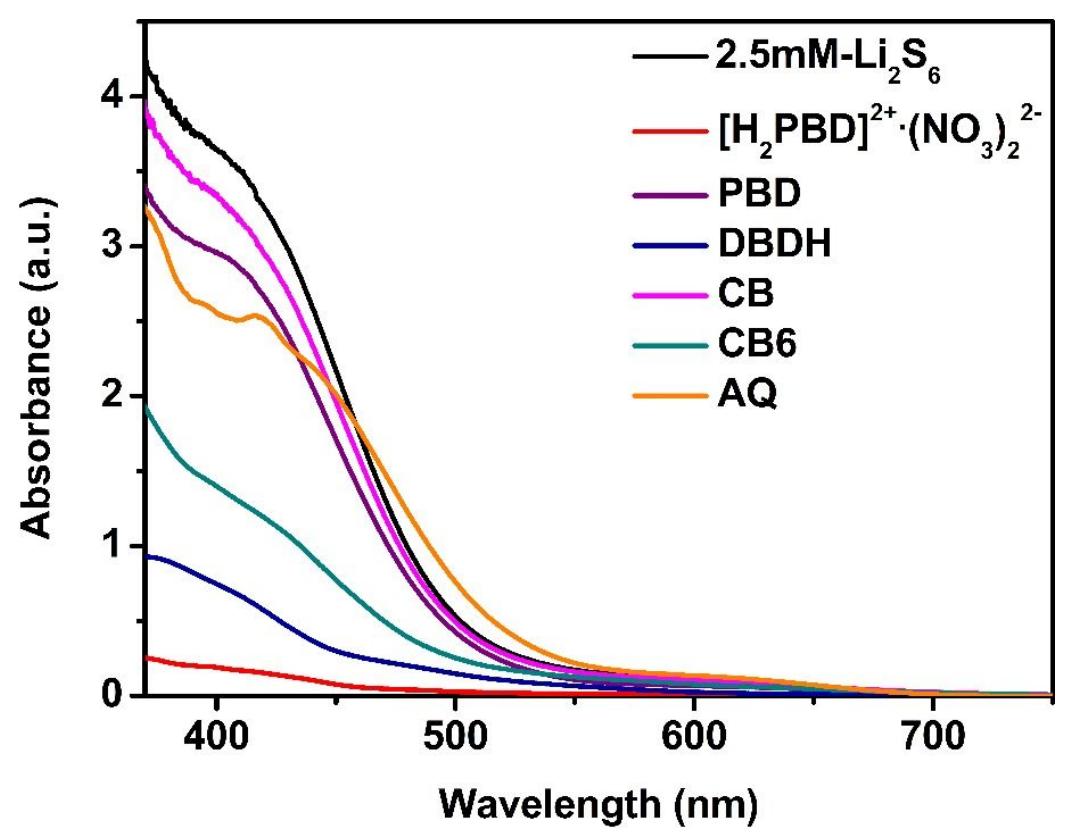

Figure S12. The UV-vis spectroscopy quantitative experiment of $5 \mathrm{mg}$ candidate materials adsorbed in $6 \mathrm{ml} \mathrm{Li}{ }_{2} \mathrm{~S}_{6}$ solution $(2.5 \mathrm{mM})$ after $48 \mathrm{~h}$. The concentration of residual PSs in the solution was determined by UV-vis adsorption at $400 \mathrm{~nm}$. 


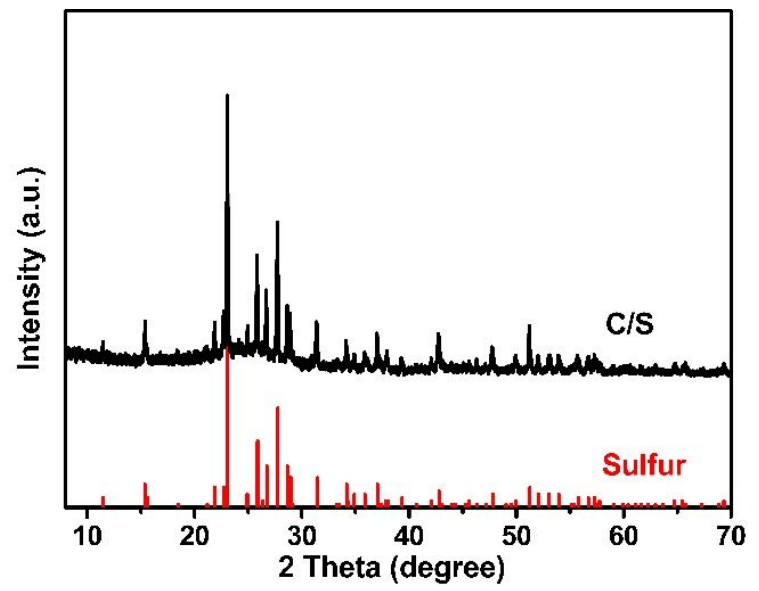

Figure S13. XRD patterns of pure $\mathrm{S}$ and $\mathrm{CB} / \mathrm{S}$ composites. 


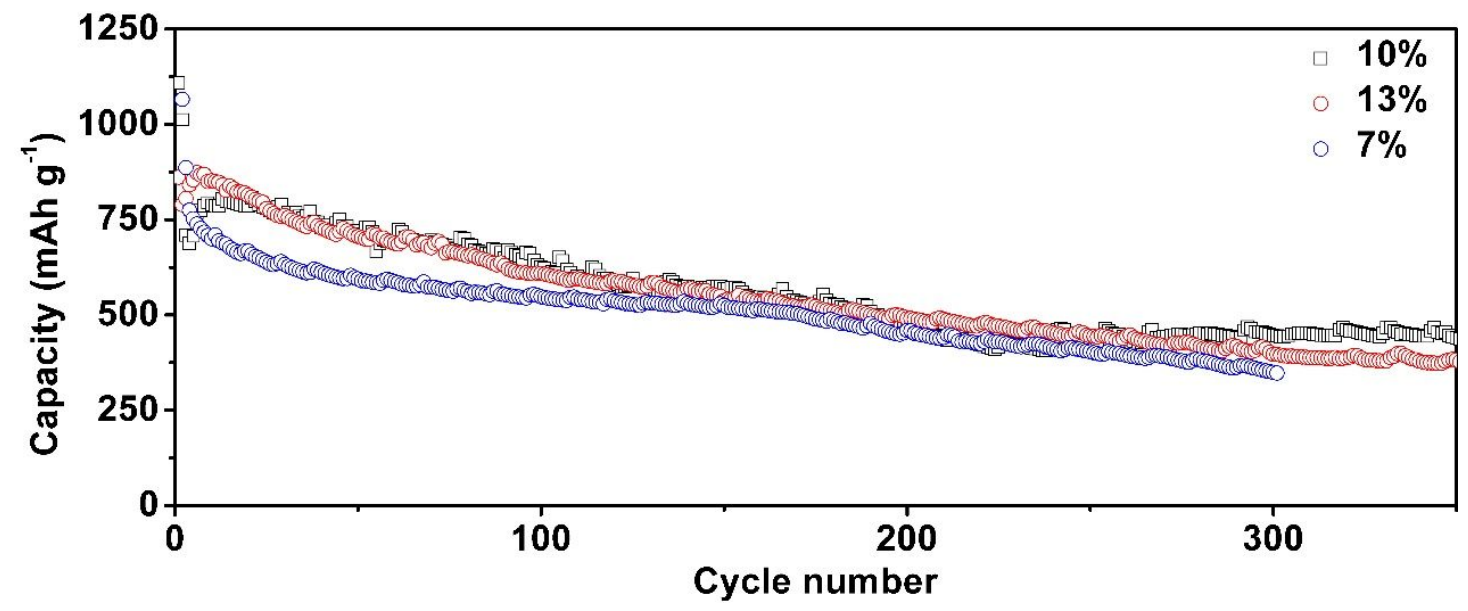

Figure S14. Performance of $\left[\mathrm{H}_{2} \mathrm{PBD}\right]^{2+} \cdot\left(\mathrm{NO}_{3}\right)_{2}{ }^{2-} / \mathrm{CB} / \mathrm{S}$ electrode at $0.5 \mathrm{C}$ under different additive content. 


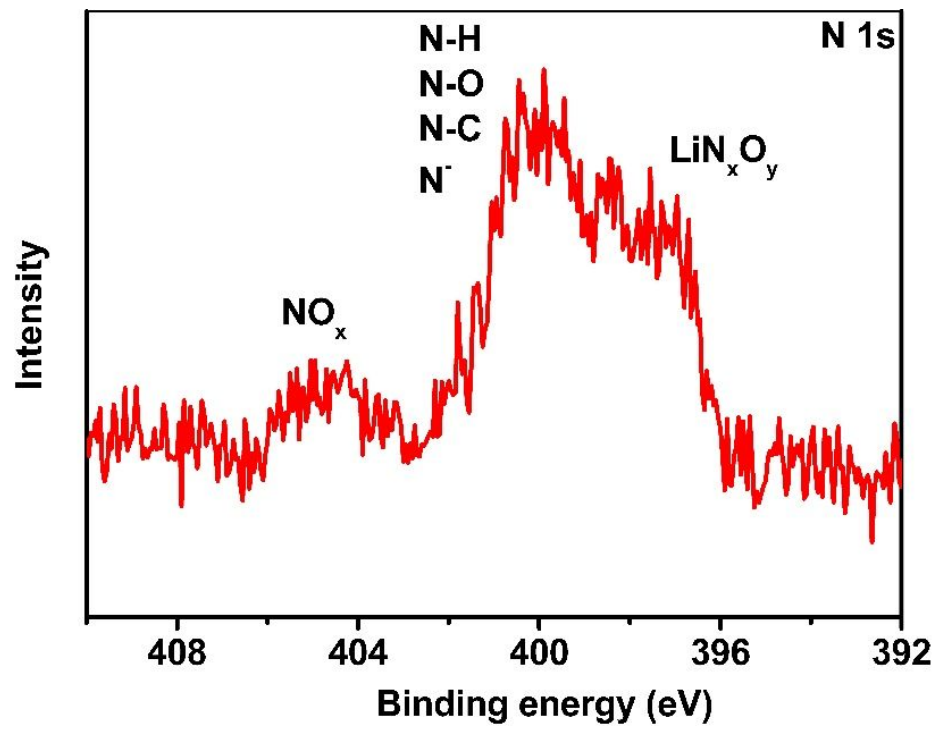

Figure S15. The XPS spectra of N 1s for Li anode surface of a cycled cell. ${ }^{[2]}$ 


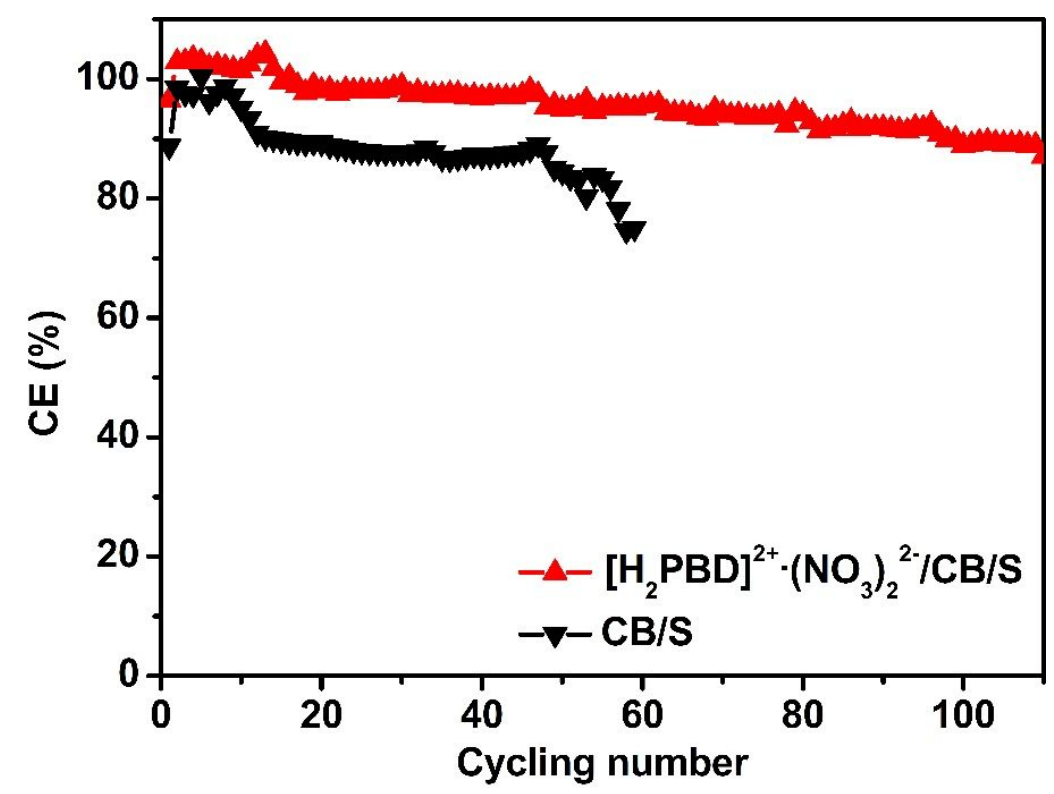

Figure S16. Cycling performance of $\left[\mathrm{H}_{2} \mathrm{PBD}\right]^{2+} \cdot\left(\mathrm{NO}_{3}\right)_{2}{ }^{2-} / \mathrm{CB} / \mathrm{S}$ and $\mathrm{CB} / \mathrm{S}$ electrodes at a current density of $0.5 \mathrm{C}$ in the absence of $\mathrm{LiNO}_{3}$ electrolyte. The electrolyte was 1.0 $\mathrm{M}$ lithium bis(trifluoromethanesulfon)imide dissolved in 1: 1 (in volume) 1,3dioxolane/dimethoxyethane. 
Table S1. The comparison of $\left[\mathrm{H}_{2} \mathrm{PBD}\right]^{2+} \cdot\left(\mathrm{NO}_{3}\right)_{2}{ }^{2-}$ with other materials adsorption for PSs.

\begin{tabular}{|c|c|c|c|c|c|c|c|}
\hline \multirow[t]{2}{*}{ Materials $^{\mathrm{a}}$} & \multirow[t]{2}{*}{$\begin{array}{l}\text { Limited PSs } \\
\text { mode }\end{array}$} & \multirow{2}{*}{$\begin{array}{l}\text { Adsorbing } \\
\text { capacity } \\
(\mathrm{g} / \mathrm{g})\end{array}$} & \multirow[t]{2}{*}{$\begin{array}{l}\text { Adsorption } \\
\text { time }\end{array}$} & \multicolumn{3}{|c|}{$\begin{array}{c}\text { Binding Energy } \\
(\mathrm{eV})\end{array}$} & \multirow[t]{2}{*}{ Ref. } \\
\hline & & & & $\mathrm{Li}_{2} \mathrm{~S}_{4}$ & $\mathrm{Li}_{2} \mathrm{~S}_{6}$ & $\mathrm{Li}_{2} \mathrm{~S}_{8}$ & \\
\hline CB6 & $\begin{array}{c}\text { Framework } \\
\text { structure/H } \\
\text { bond }\end{array}$ & 0.381 & $50 \mathrm{~min}$ & $\begin{array}{c}- \\
1.02\end{array}$ & $\begin{array}{c}- \\
1.10\end{array}$ & $\begin{array}{c}- \\
1.03\end{array}$ & 34 \\
\hline AQ & $\begin{array}{c}\text { Chemical } \\
\text { bond }\end{array}$ & 0.183 & $10 \mathrm{~min}$ & $\begin{array}{c}- \\
0.52\end{array}$ & $\begin{array}{c}- \\
0.82\end{array}$ & $\begin{array}{c}- \\
1.00\end{array}$ & 23 \\
\hline $\mathrm{CB}$ & - & 0.05 & - & - & - & - & $\begin{array}{l}\text { This } \\
\text { work }\end{array}$ \\
\hline PBD & $\begin{array}{l}\text { Chemical } \\
\text { bond }\end{array}$ & 0.116 & $48 \mathrm{~h}$ & $\begin{array}{c}- \\
0.16\end{array}$ & $\begin{array}{c}- \\
0.19\end{array}$ & $\begin{array}{c}- \\
0.17\end{array}$ & $\begin{array}{l}\text { This } \\
\text { work }\end{array}$ \\
\hline DBDH & $\begin{array}{l}\text { electrostatic } \\
\text { interactions }\end{array}$ & 0.492 & $2 \min$ & $\begin{array}{c}- \\
0.91\end{array}$ & $\begin{array}{c}- \\
0.89\end{array}$ & $\begin{array}{c}- \\
1.01\end{array}$ & $\begin{array}{l}\text { This } \\
\text { work }\end{array}$ \\
\hline $\begin{array}{l}{\left[\mathrm{H}_{2} \mathrm{PBD}\right]^{2+.}} \\
\left(\mathrm{NO}_{3}\right)_{2}{ }^{2-}\end{array}$ & $\begin{array}{l}\text { electrostatic } \\
\text { interactions/H } \\
\text { bond }\end{array}$ & 0.586 & $5 s$ & $\begin{array}{c}- \\
1.08\end{array}$ & $\begin{array}{c}- \\
1.25\end{array}$ & $\begin{array}{c}- \\
1.34\end{array}$ & $\begin{array}{l}\text { This } \\
\text { work }\end{array}$ \\
\hline
\end{tabular}

a. CB6: cucurbit[6]uril; AQ: anthraquinone.

b. References are corresponding to the main text. 
Table S2. The comparison of $\left[\mathrm{H}_{2} \mathrm{PBD}\right]^{2+} \cdot\left(\mathrm{NO}_{3}\right)_{2}{ }^{2-}$ with other additives reported for Li-S cells.

\begin{tabular}{|c|c|c|c|c|c|c|}
\hline $\begin{array}{l}\text { Sulfur } \\
\text { cathode }^{a}\end{array}$ & Additives $^{\mathrm{b}}$ & $\begin{array}{l}\text { Content } \\
\text { of } \\
\text { additives } \\
(\%)\end{array}$ & $\begin{array}{l}\text { Capacity } \\
\left(\mathrm{mAh} \mathrm{g}^{-}\right. \\
\left.{ }^{1}\right)\end{array}$ & $\begin{array}{c}\text { C } \\
\text { Rate/Cycle } \\
\text { Number }\end{array}$ & $\begin{array}{c}\text { Decay } \\
\text { rate } \\
(\%)\end{array}$ & Ref. \\
\hline $\mathrm{MPC} / \mathrm{S}$ & $\mathrm{LiCoO}_{2}$ & 3 & 715 & $1 / 150$ & 0.13 & 3 \\
\hline $\mathrm{S}$ & Mesoporous carbon & 10 & $\sim 900$ & $0.25 / 50$ & 0.14 & 4 \\
\hline $\mathrm{SCM} / \mathrm{S}$ & $\beta-\mathrm{TiO}_{2}$ & 3.6 & 750 & $1 / 200$ & 0.19 & 5 \\
\hline $\mathrm{S}$ & $\mathrm{MgO}$ & 10 & $\sim 730$ & $0.2 / 100$ & 0.15 & 6 \\
\hline $\mathrm{S}$ & SBA-15 & 9.5 & 370 & $1 / 370$ & 0.28 & 7 \\
\hline $\mathrm{GO} / \mathrm{S}$ & $\mathrm{SiO}_{2}$ & 3 & 681 & $0.1 / 150$ & 0.018 & 8 \\
\hline $\mathrm{S}$ & Chitosan & 3.5 & 646 & $1 / 100$ & 0.18 & 9 \\
\hline $\mathrm{S}$ & $\mathrm{TiO}_{2}$ & 30 & 680 & $0.2 / 100$ & 0.23 & 10 \\
\hline $\mathrm{rGO} / \mathrm{S}$ & PTMA & 10 & 950 & $0.42 / 100$ & 0.24 & 11 \\
\hline $\mathrm{rGO} / \mathrm{S}$ & CNT/IP & 20 & 715 & $1 / 310$ & 0.088 & 12 \\
\hline $\mathrm{S}$ & {$\left[\mathrm{H}_{2} \mathrm{PBD}\right]^{2+} \cdot\left(\mathrm{NO}_{3}\right)_{2}{ }^{2-}$} & 10 & 429.2 & $0.5 / 400$ & 0.099 & $\begin{array}{l}\text { This } \\
\text { work }\end{array}$ \\
\hline
\end{tabular}

a. MPC: macroporous carbon; SCM: mesoporous carbon; GO: graphene oxide; rGO: reduced graphene oxide.

b. PTMA: poly(2,2,6,6-tetramethylpiperidinyloxy-4-yl methacrylate); CNT: carbon nanotubes; IP: imidazolium-based ionic polymer. 


\section{References}

1. Wang, Q., Hou, Y., Wu, W., et al. A Study on the Structure of Yilan Oil Shale Kerogen Based on its Alkali-Oxygen Oxidation Yields of Benzene Carboxylic Acids, ${ }^{13} \mathrm{C}$ NMR and XPS. Fuel Processing Technol. 2017, 166, 30-40.

2. Barghamadi M., Best A. S., Bhatt A. I., Hollenkamp A. F., Mahon P. J., Musameh M., Rüther T. Effect of $\mathrm{LiNO}_{3}$ Additive and Pyrrolidinium Ionic Liquid on the Solid Electrolyte Interphase in the Lithium-Sulfur Battery. J. Power Sources 2015, 295, 212-220.

3. Zhang, K., Wang, L., Cai, W., Wang, C., Li, G., Li, Z., Mao, W., Qian, Y. A Novel Class of Functional Additives for Cyclability Enhancement of the Sulfur Cathode in Lithium Sulfur Batteries. Inorganic Chemistry Frontiers 2018, 5, 2013-2017.

4. Koh, J. Y., Kim, E. H., Yang, H. J., Park, M.-S., Kim, S., Jung, Y. Effects of Porous Carbon Cathode Additives on the Electrochemical Performance of Li-S Cells. J Nanosci Nanotechnol 2016, 16, 4438-4443.

5. Evers, S., Yim, T., Nazar, L. F. Understanding the Nature of Absorption/Adsorption in Nanoporous Polysulfide Sorbents for the Li-S Battery. J. Phys. Chem. C. 2012, 116, 19653-19658.

6. Ponraj, R., Kannan, A. G., Ahn, J. H., Kim, D.-W. Improvement of Cycling Performance of Lithium-Sulfur Batteries by Using Magnesium Oxide as a Functional Additive for Trapping Lithium Polysulfide. ACS Appl. Mater. Interfaces 2016, 8, 4000-4006.

7. Ji, X., Evers, S., Black, R., Nazar, L. F. Stabilizing Lithium-Sulphur Cathodes Using Polysulphide Reservoirs. Nat. Commun. 2011, 2, 325.

8. Wei, P., Fan, M., Chen, H., Chen, D., Li, C., Shu, K., Lv, C. Ternary 
Graphene/Sulfur/SiO $\mathrm{SiO}_{2}$ Composite as Stable Cathode for High Performance Lithium-Sulfur Battery. Int. J. Hydrogen Energy 2016, 41, 1819-1827.

9. Chen, Y., Liu, N., Shao, H., Wang, W., Gao, M., Li, C., Zhang, H., Wang, A., Huang, Y. Chitosan as a Functional Additive for Highperformance LithiumSulfur Batteries. J. Mater. Chem. A. 2015, 3, 15235-15240.

10. Li, Q., Zhang, Z., Zhang, K., Xu, L., Fang, J., Lai, Y., Li, J. Synthesis and Electrochemical Performance of $\mathrm{TiO}_{2}-$ Sulfur Composite Cathode Materials for Lithium-Sulfur Batteries. J. Solid State Electrochem. 2013, 17, 2959-2965.

11. Chen, H., Wang, C., Dai, Y., Ge, J. Lu, W., Yang, J., Chen, L. In-Situ Activated Polycation as a Multifunctional Additive for Li-S Batteries. Nano Energy 2016, $26,43-49$.

12. Cheng,Z., Pan, H., Xiao, Z., Chen, D., Li, X., Wang, R. Electrostatic Trapping of Polysulfides Enabled by Imidazolium-Based Ionic Polymers for HighenergyDensity Lithium-Sulfur Batteries. J. Mater. Chem. A. 2018, 6, 7375-7381. 